Bundesgesundheitsbl -

Leitthema:Reisemedizin

S. Ley ${ }^{1} \cdot$ U. Quast ${ }^{1} \cdot$ S. Reiter ${ }^{2}$

${ }^{1}$ Deutsches Grünes Kreuz, Marburg $\cdot{ }^{2}$ Robert Koch-Institut, Berlin

\title{
Qualifizierte reise- medizinische Beratung
}

\section{Bedeutung, Anforderungen, Handlungsbedarf}

\section{Zusammenfassung}

Die reisemedizinische Beratung und Betreuung gewinnt angesichts der zunehmenden weltweiten Mobilität und der ebenfalls ungebrochenen Reiselust der Deutschen immer mehr an Bedeutung und wird durch neue Impfstoffe, veränderte Reisegewohnheiten und veränderte epidemiologische Ausgangsbedingungen bei einzelnen Erregern immer komplexer. Die qualifizierte reisemedizinische Beratung setzt besonderes Fachwissen und aktuelle, differenzierte Informationen über die Reiseländer voraus. Die Qualität der vielfältigen reisemedizinischen Beratungsangebote weicht in Deutschland stark voneinander $a b$ und für den Reisenden ist oft nicht erkennbar, wer auf diesem Gebiet kompetent berät. Lösungsansätze für eine Verbesserung der Qualität und Transparenz in der reisemedizinischen Beratung bestehen u.a.in der Weiterentwicklung von Qualitätsstandards, in der Zertifizierung sowie öffentlichen Bekanntmachung qualifizierter Beratungsstellen und in einer engeren Zusammenarbeit von Reisebüros, Apotheken und Ärzten. er vorliegende Beitrag basiert auf der Zusammenfassung und Aktualisierung ausgewählter Vorträge des Workshops „Reisemedizin in Deutschland Anforderungen, Defizite und Perspektiven“, der am 13./14. Mai 1998 in Berlin stattfand und über dessen wichtigste Ergebnisse im Epidemiologischen Bulletin 25/98 bereits kurz berichtet wurde.

\section{Die Bedeutung qualifizierter reisemedizinischer Beratung}

Mit der zunehmenden weltweiten Mobilität gewinnt die Reisemedizin immer mehr an Bedeutung. Mehr als 500 Millionen internationale Flugankünfte werden jährlich verzeichnet, für das Jahr 2000 sind 673 Millionen internationale Ankünfte prognostiziert [1]. Der Trend zum Reisen ist auch bei den Deutschen ungebrochen. 1998 gaben die Bundesbürger rund 83 Milliarden DM im Ausland aus. Statistisch gesehen reist jeder zweite Deutsche einmal im Jahr ins Ausland und jeder 20. Deutsche einmal jährlich ins außereuropäische Ausland, rund 5 Millionen Bundesbürger verbringen ihren Urlaub in den Tropen. Für 1999 erwartet die deutsche Touristikbranche einen Umsatzzuwachs von 5\%.

$\mathrm{Zu}$ den beliebtesten Reiseländern gehören nach wie vor Spanien, Italien, Österreich, die Türkei und Griechenland, aber auch Tunesien, Marokko und Ägypten. Reisestil und Reiseziele haben sich in den letzten Jahren deutlich verändert. Der traditionelle Badeurlaub ist immer noch beliebt, wird aber zunehmend von anderen Tourismusformen wie Studien-, Abenteuer-, Kultur-, Trekking- und Erlebnisreisen abgelöst. Auch die Reiseziele sind spektakulärer geworden, und die Zahl der Fernreisen nimmt kontinuierlich zu. Die medizinische Reiseberatung muß damit eine neue $\mathrm{Di}$ mension erreichen. Selbstverständlich bestehen auch bei einer Pauschalreise oder einem Badeurlaub gewisse gesundheitliche Risiken - ein Reisender, der eine Trekkingtour durch Indien plant, muß aber viel umfassender und über wesentlich mehr gesundheitliche Gefahren beraten werden als ein Urlauber, der eine Pauschalreise an die Costa Brava macht. Die qualifizierte reisemedizinische Beratung und Betreuung gewinnt nicht nur an Bedeutung, sie wird durch neue Impfstoffe, veränderte Reisegewohnheiten und veränderte epidemiologische Ausgangsbedingungen bei einzelnen Erregern auch immer komplexer.

Die in Tabelle 1 angegebenen Zahlen belegen, wie wichtig es ist, eine dem Reisestil und Reiseziel angepaßte gesundheitsbezogene Beratung vorzunehmen.

\section{Dr. Sigrid Ley}

Deutsches Grünes Kreuz, Im Kilian, Schuhmarkt 4, D-35037 Marburg 
Bundesgesundheitsbl -

Gesundheitsforsch - Gesundheitsschutz

1999 - 42: 377-380 @ Springer-Verlag 1999

S. Ley · U. Quast · S. Reiter

Qualified medical travel advice importance, requirements, proposals for action

\section{Summary}

Travel medicine in Germany is getting more important, but also more complex in view of increasing worldwide tourism, the unbroken keenness of Germans to travel, new vaccines, changed travel habits and the altered epidemiological situation of several infectious agents. Qualified health information requires extensive specialized knowledge and up-todate, differentiated information about travel destinations. The quality of the manifold counselling services varies considerably, and it is often difficult for the traveller seeking medical advice to make judgements about the quality of counselling. Possible solutions for the improvement of quality and transparancy in this field are seen - amongst others - in the further development of quality standards, the certification and publication of qualified information and in a closer cooperation between travel agencies, pharmacies and physicians.

\section{Leitthema: Reisemedizin}

\begin{tabular}{lc} 
Tabelle 1 & \\
Geschätzte Zahl der Infektionen nach Tropenreisen bezogen \\
auf 100000 Touristen pro Monat, Angaben der WHO \\
Reisediarrhoe & $3000-70000$ \\
Malaria & 2400 \\
Akute fieberhafte Erkrankungen der Atemwege & 1300 \\
Hepatitis A (Hoteltouristen) & 300 \\
Gonorrhoe & 200 \\
Biß durch tollwutgefährdete Tiere & 130 \\
Hepatitis B (längerer Aufenthalt) & 80 \\
Typhus (Indien, Nord- und Westafrika) & 30 \\
HIV-Infektion & 10 \\
Typhus & 3 \\
Poliomyelitis (asymptomatisch) & 2 \\
Legionellosen (Mittelmeerraum) & 1 \\
Cholera & 0,2 \\
Paralytische Poliomyelitis & 0,07 \\
\hline
\end{tabular}

Quelle: Reisen und Gesundheit 1998, Verlag im Kilian
Bei Reisen in tropische Länder ist die Hepatitis A mit einem durchschnittlichen Erkrankungsrisiko von 0,3\% pro Monat die häufigste impfpräventable Krankheit, bei Rucksacktouristen erhöht sich das Risiko auf $2 \%$.

In Südamerika haben im Jahr nach „El niño” die Malariaerkrankungen um $36 \%$ zugenommen, durch ökologische und klimatische Veränderungen sind auch das Dengue-Fieber und zahlreiche andere Infektionskrankheiten auf dem

„Zwar gibt es keinen 100\%igen
Schutz vor tropischen Erkrankungen,
eine rechtzeitige individuelle und
ausführliche Beratung vor Reisebe-
ginn kann das Risiko einer Erkran-
kung jedoch erheblich reduzieren."

Vormarsch. Das Risiko, sich bei Auslandsaufenthalten mit Hepatitis B zu infizieren, besteht hauptsächlich bei Langzeitaufenthalten oder risikoreichem Verhalten wie ungeschützten Sexualkontakten oder intravenösem Drogenkonsum. Die Gefahr einer Infektion mit Tollwut wird von Langzeitreisenden und Ruck- sacktouristen in südostasiatischen Ländern oft unterschätzt.

In Deutschland sind neben der Hepatitis A die Malaria, die Shigellose und andere Darminfektionen die häufigsten importierten Infektionskrankheiten. Die Anfang 1998 im Vergleich zum Vorjahreszeitraum fast doppelt so hohen Malariazahlen sind überwiegend auf die Überschwemmungen in Kenia im November/Dezember 1997 zurückzuführen. 1998 wurden dem Robert Koch-Institut (RKI) insgesamt 996 Malaria-Infektionen gemeldet, 20 Personen starben daran. Allein im Bernhard-Nocht-Institut in Hamburg werden jährlich ca. 1000 Dengue-Fälle diagnostiziert. Bei 20\% handelt es sich um Zweitinfektionen, die sehr oft schwerer als die Erstinfektionen verlaufen. Während bei Erwachsenen tödliche Verläufe nicht sehr häufig sind, ist die Sterblichkeit bei Kindern hoch. Kinder sollten wegen des Enhancement-Risikos daher nicht einer erneuten Infektionsgefahr ausgesetzt werden. Zwar gibt es keinen 100\%igen Schutz vor tropischen Erkrankungen, eine rechtzeitige individuelle und ausführliche Beratung vor Reisebeginn kann das Risiko einer Erkrankung jedoch erheblich reduzieren. 


\section{Reisemedizinische Beratungs- angebote in Deutschland}

In der Bundesrepublik Deutschland bietet eine Vielzahl von öffentlichen und privaten Institutionen reisemedizinische Beratung und Informationensmaterialien für die Bevölkerung an. Die Angebote reichen von allgemeinen und länderspezifischen Broschüren, Merkblättern, Presseveröffentlichungen, von telefonischer und computergestützer Beratung und Videos bis zur individuellen reisemedizischen Beratung und der Durchführung von Schutzimpfungen bei niedergelassenen Ärzten, Tropeninstituten, Gesundheitsämtern, betriebs-, hafen- und flughafenärztlichen Diensten. Für die reisemedizinische Beratungspraxis wurden Computerprogramme und Informationsmaterialien entwickelt; viele Institutionen bieten Fortund Weiterbildung für Ärzte und Apotheker auf diesem Gebiet an. Zahlreiche Studien belegen jedoch, daß die Qualität der Beratungsangebote sehr unterschiedlich ist, die reisemedizinischen Empfehlungen voneinander abweichen und teilweise erhebliche Wissensdefizite bei den Beratern bestehen. So ist laut einer Studie der Stiftung Warentest jeder vierte Deutsche vor einer Reise schlecht beraten [2]. Auch die Transparenz der Beratungsangebote läßt in Deutschland zu wünschen übrig, da für den Reisenden nicht erkennbar ist, wer kompetente Beratung auf diesem Gebiet anbietet.

Bei der reisemedizinischen Beratung spielen die niedergelassenen Ärzte eine wichtige Rolle: Laut einer Umfrage des RKI mit Schwerpunkt bei den unter 27jährigen Rucksacktouristen stand der Hausarzt bei der Reiseinformation ganz im Vordergrund [3]. Die Rolle der Apotheker und Reisebüros darf nicht unterschätzt werden, doch stellt sich auch hier die Frage der Qualifikation.

Die Reiseveranstalter sind mit Ausnahme der Gelbfieberimpfbestimmungen nicht zur Aufklärung über gesundheitliche Risiken im Reiseland verpflichtet. Einige Reiseveranstalter haben ihre gesundheitlichen Informationsangebote in den vergangenen Jahren jedoch beträchtlich erweitert und verbessert. Die
Rolle der Reiseveranstalter besteht vor allem in einer ersten Information ihrer Kunden auf im Reiseland vorkommende Erkrankungsrisiken und der Motivation zu einer fachmedizinischen Beratung.

\section{Anforderungen an eine qualifizierte reisemedizinische Beratung}

Das Verständnis der Reisemedizin hat sich in den letzten Jahren grundlegend verändert: Stand früher die Verhütung der Einschleppung von Krankheiten (Vorsorge öffentlicher Gesundheit), die Feststellung der Tropentauglichkeit und die Behandlung von importierten Krankheiten im Vordergrund, so kommt heute ärztlicher Beratung, Betreuung und Gesundheitsschutz vor beruflichen und privaten Reisen die größere Bedeutung zu (individuelle Vorsorge).

\section{"Die reisemedizinische Beratung setzt besonderes Fachwissen und aktuelle, differenzierte Informatio- nen über die Reiseländer voraus."}

Eine qualifizierte Beratung muß ausführlich auf die länderspezifischen (Region, Saison) und individuellen Krankheitsrisiken eingehen, die Sondersitutation einzelner Reisender beachten sowie die Dauer und Art der Reise berücksichtigen. Neben Informationen über Impfungen, die für das Reiseland vorgeschriebenen bzw. empfohlenen sind (inkl. der Überprüfung des Impfstatus für Diphtherie, Tetanus und Poliomyelitis), muß auch über weitere Prophylaxemaßnahmen bei nicht impfpräventablen Krankheiten, die durch Insekten oder Nahrungsmittel und Getränke übertragbar sind, mögliche Unfallgefahren, klimatische Belastungen sowie Sonnenund Mückenschutz aufgeklärt werden.

\section{Sonstige Prophylaxe}

Eine erweiterte Aufklärung zur sonstigen Prophylaxe beinhaltet Themen wie: D Hitze und Feuchtigkeit

- Sonnenschäden

- Kälteschäden, auch Tag-NachtSchwankungen
D Tier-, Schlangen- und Skorpionbisse

- Krankheiten, die durch Baden oder Im-Wasser-Waten in Binnengewässern übertragen werden

D Krankheiten, die beim Baden im Meer entstehen

D Krankheiten, die beim Barfußgehen drohen

- Gesundheitsgefahren bei großen Höhenunterschieden

D Unfälle

- Sexuell übertragbare Erkrankungen

Darüber hinaus muß abgeklärt werden, ob eventuell eine Sondersituation für den Reisenden oder seine Mitreisenden besteht. Hierzu zählen vor allem Reisen von Senioren, die aufgrund des nachlassenden Immunsystems ein erhöhtes Risiko für Infektionskrankheiten besitzen, Reisen von Schwangeren, chronisch Kranken und Reisen mit Kindern.

\section{Impfung von Schwangeren}

Bei einer Schwangerschaft wären folgende Fragen abzuklären bzw. anzusprechen:

D In welchem Schwangerschaftsmonat befindet sich die Patientin?

- Verlief die Schwangerschaft bisher komplikationslos?

D Erst- oder Multipara?

D Muß die Patientin besondere Medikamente einnehmen, die Vorsichtsmaßnahmen oder Kontrollen erfordern?

D Ist die Reise zu verantworten?

Dind Impfungen erforderlich?

- Ist eine Malariaprophylaxe erforderlich, ggf. besondere Prophylaxe?

- Müssen zur Malariaprophylaxe Standby-Medikamente mitgenommen werden?

D Ist die Schwangere flugtauglich?

- Sind Hinweise auf sonstige Risiken für die Schwangerschaft erforderlich?

\section{Chronisch Kranke}

Bei chronischen Erkrankungen wären folgende Themen anzusprechen:

D Unter welcher chronischen Erkrankung leidet der Patient?

D Ist er dennoch reisefähig?

D Ist seine chronische Erkrankung medikamentös gut eingestellt? 
D Hat der Patient das Medikament (und evtl. Spritzen) in ausreichender Menge vorrätig?

D Ist der Patient flugtauglich?

D Gibt es Impfungen, die notwendig, aber bei dem Patienten grundsätzlich kontraindiziert sind?

D Sollte der Patient zusätzliche Impfungen (z. B. Influenza, Pneumokokken) erhalten?

D Welche Malariamittel kann der Patient einnehmen?

D Gibt es Wechselwirkungen mit anderen Medikamenten?

D Sind Hinweise auf sonstige Risiken durch die Medikamente erfolgt?

D Sind Hinweise auf sonstige Risiken durch die Reise erfolgt?

\section{Malaria}

Beim Schutz vor Malaria gibt es drei Möglichkeiten: die Expositionsprophylaxe, die Chemoprophylaxe und die Therapie. Das Risiko einer Malariaerkankung ist gemessen am Übertragungspotential und an Art und Resistenzlage des Parasiten in den Tropen sehr unterschiedlich ausgeprägt. Die „ABC-Karte“ der WHO wird oft falsch interpretiert. So benötigen beispielsweise über $90 \%$ der Reisenden nach Thailand keine Malariaprophylaxe, obwohl Thailand im C-Gebiet liegt. Das Risiko, sich in Touristengebieten Thailands in einer Nacht infektiöse Mückenstiche zuzuziehen, liegt bei o,003\%, dagegen ist das Infektionsrisiko in Tansania (ebenfalls ein C-Gebiet) bei 8-10\% infektiöser Anopheles-Mücken extrem hoch.

\section{„Für eine gute reisemedizinische Beratung und Betreuung sind viel Zeit und Wissen erforderlich."}

\section{Nutzen-Risiko-Abwägung}

Um unter einer vertretbaren Nutzen-Risiko-Abwägung zu einer individuellen Vorbeugeempfehlung zu kommen, muß der Berater die regionalen Bedingungen des Reisegebietes, die Reisesaison und die klimatischen Verhältnisse berücksichtigen und mit der Aufenthaltsdauer, dem Reisestil, möglichen chronischen Krankheiten, der Zugehörigkeit zu einer Risikogruppe (z.B. Kinder, Schwangere, ältere Menschen, Immundefiziente), der ärztlichen Versorgung vor Ort und der Compliance des Reisenden korrelieren. Notwendig ist auch der Hinweis, daß bei Auftreten einer verdächtigen Symptomatik, vor allem bei Fieber während und nach einem Aufenthalt in einem Malariagebiet, ein Arzt aufgesucht werden sollte.

Diese Beispiele, die keinen Anspruch auf Vollständigkeit erheben und beliebig ergänzt werden können, zeigen, $\mathrm{da}$ für eine gute reisemedizinische $\mathrm{Be}$ ratung und Betreuung viel Zeit und Wissen erforderlich sind, das zunächst erworben und dann regelmäßig aufgefrischt werden muß. Ein detaillierter reisemedizinischer Anamnesebogen für niedergelassene Ärzte, der diese Aspekte differenzierter reisemedizinischer Beratung berücksichtigt, könnte auch als Qualitätssicherungsinstrument eingesetzt werden.

\section{Lösungsansätze zur Verbesserung der reisemedizinischen Beratung}

Insgesamt müssen der Weg des Reisenden zu den beratenden Stellen und die Transparenz und Qualität der Beratungsangebote verbessert werden. Eine effektive Prävention auf diesem Gebiet beinhaltet auch das Zugehen auf die Reisenden und verstärkte Werbung für reisemedizinische Beratung.

\section{Lösungsansätze zur Verbesserung der reisemedizinischen Beratung bestehen vor allem:}

D In einer verstärkten Zusammenarbeit an der Basis („Reisemedizinisches Dreieck“: Reisebüro, Apotheke, Arzt),

D In der Harmonisierung reisemedizinischer Beratungsschemata

D In der Erarbeitung von Qualitätsstandards für die reisemedizinische Beratung und Betreuung sowie der Zertifizierung der Qualfikation,

D In der öffentlichen Bekanntmachung qualifizierter Beratungsstellen,

D In einer stärkeren Einbeziehung von betriebsärztlichen/überbetrieblichen arbeitsmedizinischen Diensten

D In der Vernetzung der relevanten medizinischen Versorgungseinrichtungen und Fachinstitutionen und

D In einer engeren Zusammenarbeit mit den Reiseveranstaltern und Reisebüros.

Durch die weltweit zunehmende Bedeutung des Tourismus müssen neben einer verbesserten reisemedizinischen Beratung und Betreuung auch die Diagnostik und Surveillance reisebedingter Krankheiten verbessert und die internationale Kooperation gestärkt werden.

\section{Literatur}

1. Cetron M, Keystone J, Shlim D, Steffen R (1998) Travelers' Health. In: Emerging Infectious Diseases. 4 (3): 405

2. Stiftung Warentest (1996) Haben Schlangen Tollwut? In: Test 10/96

3. Tiemann F, Grote H, Grote S (1998) Impfschutz und Infektionsprophylaxe bei Fernreisenden. Eine schriftliche Befragung von Fernreisenden auf dem Rückflug aus ausgewählten Reisegebieten. In: InfFO II/98, S 18-22 